\title{
On A New Interconnection Network for Large Scale Parallel Systems
}

\author{
Nibedita Adhikari \\ Assistant professor and Head \\ Department of Computer Science \& Engineering \\ Purushottam Institute of Engineering \&Technology \\ Mandikudar, 770034 \\ Rourkela, Orissa, India
}

\author{
C. R. Tripathy \\ Professor, Department of CSE \\ Veer Surendra Sai University of Technology \\ Burla, Sambalpur, Orissa, India
}

\begin{abstract}
This paper proposes a new cube based topology called the Folded Metacube (FMC). The new topology has attractive features such as reduced diameter, cost and improved broadcast time in comparison to the Metacube. Two separate routing algorithms one-to-one and one-to-all broadcast are proposed for the new network. Performance analysis such as cost effectiveness, time-cost-effectiveness and reliability of the new topology are carried out. The proposed network exhibits noticeable improvement in terms of the topological parameters and is superior to the other existing cube based networks.
\end{abstract}

\section{General Terms}

Parallel Computing, Performance analysis

\section{Keywords}

Interconnection network; Folded hypercube; Metacube, Routing, Performance analysis, Reliability analysis

\section{INTRODUCTION}

The cube based networks due to their splendid interconnection structures with large bandwidth, logarithmic diameter and high degree of fault tolerance, have received over the past few years $[6,7,9$,$] . Many interconnection networks such as trees and$ multidimensional meshes can be embedded in the cube. Extensive research has resulted in several variations of the cube such as Hypercube [12], Folded hypercube[4], Crossed cube [8],Folded crossed cube [1], Dual-cube [10] Metacube [11] and Folded dualcube [2].

Metacube Network is a very large scale parallel system as compared to the Hypercube. It links millions of nodes with a small node degree. The Metacube network called as $\mathrm{MC}(\mathrm{k}, \mathrm{m})$ is a two level cube structure. A Metacube network $\mathrm{MC}(\mathrm{k}, \mathrm{m})$ can connect $2^{k+m 2^{k}}$ nodes with $(\mathrm{k}+\mathrm{m})$ links per node. It contains $2 \mathrm{k}$ classes. Each class contains $2 \mathrm{mh}+\mathrm{k}$ clusters and each cluster contains $2 \mathrm{~m}$ nodes. $\mathrm{MC}(1, \mathrm{~m})$ is the Dualcube [10]. In $\mathrm{MC}(2,2), \quad \mathrm{m}$ links are used within the clusters to construct an $\mathrm{m}$ cube and $\mathrm{k}$ links are used to connect a node in a cluster of the other class. There is no link between the clusters of the same class. If two nodes are in the same cluster or in two clusters of different classes then, the distance between them is equal to the hamming distance, otherwise it is the hamming distance plus two [10].

The Folded hypercube [4] of dimension $n$ called as FHC(n) is constructed from the $n$-cube by connecting each node to the unique node that is farthest from it. Thus, FHC(n) is a regular network of degree $(n+1)$. The Hypercube of degree 3 can also be converted to FHC (3) network by introducing cross links between the farthest nodes. The aim is to reduce the diameter. Thus for large scale parallel systems efficient and cost effective inter processor communication is always essential.

Recently, the authors in [2] have proposed a new interconnection network topology called Folded dualcube, FDC $(1, \mathrm{~m})$ for parallel systems. However the said network consists of only two classes of clusters. A variation of the Metacube network has been proposed in [3], which inherits some of the useful properties of the Metacube and Folded Hypercube. Some of the topological features are derived with an aim to improve the diameter, cost and broadcast time while connecting to a large number of nodes.

The current work studies the topological properties and analyzes the performance of the Folded Metacube FMC $(\mathrm{k}, \mathrm{m})$. Different performance parameters are evaluated and compared with those of the Metacube and Folded Hypercube networks. The following section presents the topological properties of the new network. Section 3 of the paper proposes two routing algorithms. In section 4 performance analysis and comparison is done. Results are presented in Section 5. Section 6 concludes the paper.

\section{THE PROPOSED TOPOLOGY}

This section proposes a new interconnection topology: Folded Metacube. Before that we discuss the topological features of two important cube-based topologies of interest: Metacube topology and Folded Hypercube topology. To describe the network structure the graph theoretical model is used.

\subsection{The Metacube Topology}

As already discussed in section 1 of this paper a Metacube topology has two parameters $\mathrm{k}$ and $\mathrm{m}$. The Metacube network $\mathrm{MC}(\mathrm{k}, \mathrm{m})$ can be modeled as a graph $\operatorname{Gr}(\mathrm{V}, \mathrm{E})$ with $|V|=$ $2^{m h+k}$ and $|E|=r 2^{m h+k-1}$ where $\mathrm{h}=2^{k}$ and $\mathrm{r}=\mathrm{m}+\mathrm{k}$. Each node $\mathrm{u} \in \mathrm{V}, \mathrm{a}(\mathrm{u})$ denotes the $(\mathrm{mh}+\mathrm{k})$ bit binary address which is divided into three parts: a k-bit class address, an m(h-1) bit cluster address and m-bit node address. So Gr contains $2^{k}$ number of classes. Each class will contain $2^{m h-1}$ clusters and each cluster contains $2^{m}$ number of nodes. Next $\|a(u)\|$ denotes the number of 1's that is the hamming weight 
in the binary bit representation of $a(u)$. Then $e=(u, v) \quad E$, iff $=1$, where $\oplus$ is the XOR operator giving the hamming distance between nodes $u$ and $v$. So degree $(\mathrm{u})=\mathrm{r}$ for every node $\mathrm{u} \quad \mathrm{V}$. The structure of MC network of dimension 4 that is G4 is shown in Fig. 1 below. The cluster is a 2-cube and there are 4 classes. The clusters in a square belong to a single class and clusters of same class are not connected.

\subsection{The Folded Hypercube}

The folded hypercube [4] of dimension n, FHC(n) is constructed from the $\mathrm{n}$-cube by connecting each node to the unique node that is farthest from it. Thus FHC(n) is a regular network of degree $(n+1)$. The number of edges is increased by a factor equal to (total number of nodes/2). With increased number of links the diameter is reduced to half as compared to the diameter of general hypercube. The hypercube of degree 3 is converted to FHC(3) network as shown in Fig. 2.

\subsection{The Folded Metacube FMC}

The Folded Metacube, constructed from Metacube $G_{r}(V, E)$ as shown in Fig.1, by connecting each node to a node farthest from it similar to FHC as shown in Fig. 2.

$$
\begin{aligned}
& r=m+k . \\
& |V|=2^{m h+k},|E|=r .2^{m h+k-1}
\end{aligned}
$$

The Folded Metacube can be represented as a graph $F_{r}(V, \quad)$ as shown in Fig. 3, with the same set of vertices as in $\mathrm{G}_{\mathrm{r}}$ and with the edge set that is a super set of E. In Fig.3 some of the complementary links are shown for better clarity. Now

$$
\begin{aligned}
& =|E|+(\text { Total no of nodes }) / 2 \\
= & r \cdot 2^{m h+k-1}+|V| / 2=(r+1) 2^{m h+k-1}
\end{aligned}
$$

The $\mathrm{G}_{\mathrm{r}}$ is a spanning sub graph of $\mathrm{F}_{\mathrm{r}}$ and $\mathrm{e}(\mathrm{u}, \mathrm{v}) \in$, iff $\|a(u) \oplus a(v)\|=1$ or $(m h+k)$ where $\oplus$ is the $\mathrm{XOR}$ operator giving hamming distance. $\|a(u) \oplus a(v)\|=m h+k$ because each node is connected to its farthest node using the dotted links.

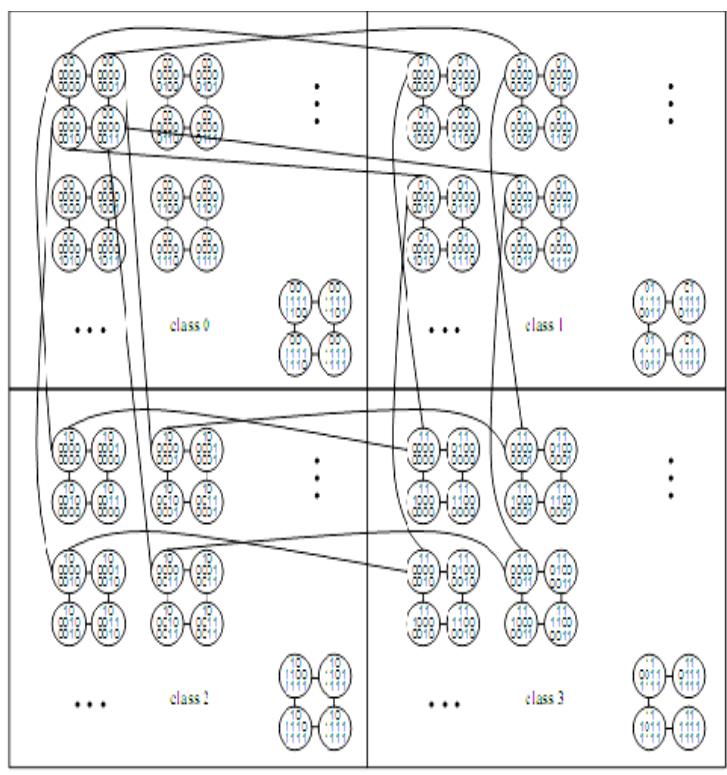

Figure 1: Metacube $\mathrm{MC}(2,2)$ or $\mathbf{G}_{\mathbf{4}}$

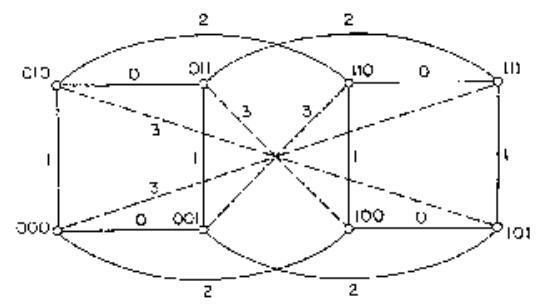

Figure2: Folded Hypercube FHC(3)

That means every node $\mathrm{u}$ in $F_{r}$ is connected to $r$ nodes with hamming distance 1 and one node ate hamming distance $(\mathrm{mh}+\mathrm{k})$.

\section{Topological Properties of Folded Metacube}

This section describes the topological parameters of the Folded Metacube. Let $G$ and $F$ represent the topologies of Metacube and the Folded Metacube respectively.

Proposition 3.1. The degree of $F_{r}$ is $(r+1)$.

Proof: In $\mathrm{G}_{\mathrm{r}}$, that is the $\mathrm{MC}$ network the node degree is $\mathrm{r}$. But in FMC, as new complementary edges are introduced to connect each node to its farthest node, so the node degree is increased by 1 . Hence degree of $F_{r}$ is

$$
\operatorname{Deg}\left(\mathrm{F}_{\mathrm{r}}\right)=(r+1) \text {. }
$$




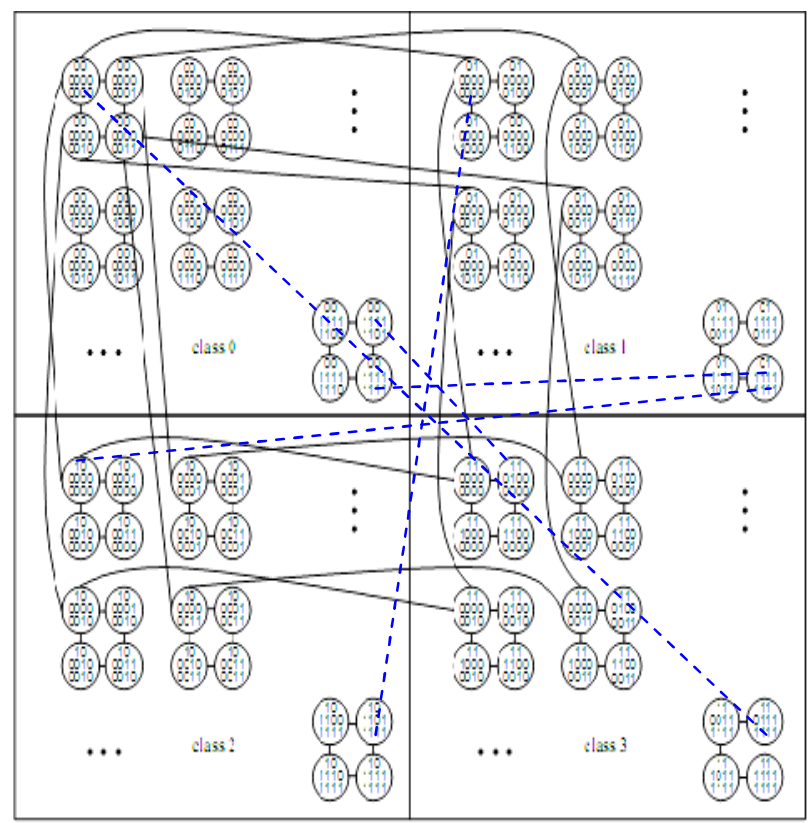

Figure 3: Folded Metacube of dimension 4, FMC(2,2) or $\mathrm{F}_{4}$

Proposition 3.2. The total number of nodes in $F_{r}$ is

Proof: In FMC network the number of nodes is same as that of MC network. Hence the result.

Proposition 3.3: The total number of edges in $F_{r}$ is given by $2^{m 2^{k}+k-1}(r+1)$.

Proof: In $\mathrm{G}_{\mathrm{r}}$ the total number of edges is given by

In the proposed network $\mathrm{F}_{\mathrm{r}}$ the number of complementary edges added is

$$
\left|\frac{V}{2}\right|=\frac{2^{m h+k}}{2}
$$

So, the total number of edges is given by

$$
\begin{gathered}
E^{\prime}=E+\left|\frac{V}{2}\right| \\
=(m+k) 2^{m h+k-1}+\frac{2^{m h+k}}{2}=(\mathrm{m}+\mathrm{k}+1) 2^{m h+k-1} \\
\text { Hence } E^{\prime}=(r+1) 2^{m h+k-1}
\end{gathered}
$$

Proposition 3.4. The node connectivity of $\mathrm{F}_{\mathrm{r}}$ is $(r+1)$.

Proof: Every node with $(m h+k)$ bit address $\mathrm{a}(\mathrm{u})$ in $\mathrm{F}_{\mathrm{r}}$ is connected to $r$ nodes at a hamming distance 1 and one node at hamming distance $(m h+k)$. So degree of $\mathrm{F}_{\mathrm{r}}$ is $d_{F}(u)=r+1$ and $\mathrm{F}_{\mathrm{r}}$ is a regular network of degree $(r+1)$.

Proposition 3.5. The number of node disjoint paths between any two nodes of $\mathrm{F}_{\mathrm{r}}$ is $(r+1)$.

Proof: Since every node in $\mathrm{F}_{\mathrm{r}}$ has $(r+1)$ neighbors so it is necessary to remove at least $(r+1)$ nodes to disconnect $\mathrm{F}_{\mathrm{r}}$. Hence the number of nodedisjoint paths $n \mathrm{~F}_{\mathrm{r}}$ is $(r+1)$.
Proposition 3.6. Diameter of $F_{r}$ is $2 r-2$.

Proof: Let $s \& t \in V$ in $F_{r}$ differ in $p$ bit positions. Then in Folded Metacube, if $\mathrm{s}$ and $\mathrm{t}$ are in the same cluster, then maximum distance is given by $\mathrm{d}^{\prime}(\mathrm{s} \mathrm{t})=\min .(p,(m h+k)-p+1)$. If $\mathrm{s}$ and $\mathrm{t}$ are of different class, then $\mathrm{d}^{\prime}(\mathrm{s} \mathrm{t})=\min .(p, \quad(m h+k)-$ $p+1)$. If $\mathrm{s}$ and $\mathrm{t}$ are in different clusters of same class, then

$$
\mathrm{d}^{\prime}(\mathrm{s} \mathrm{t})=\min \cdot(p+2, \quad(\operatorname{mh}+k)-p+1)
$$

Maximum distance in MC occurs when the node addresses differ in cluster and node address bits, but the class address bits remaining the same, i.e $2^{k}(m+1)$. The maximum distance in FMC for the same two nodes, is $2 r-2$ where $r=m+k$.

Proposition 3.7. The cost of the FMC network is $(r+1)(2 r-2)$.

Proof: In general the cost of an interconnection network is defined as the product of node degree and network diameter. In $\mathrm{F}_{\mathrm{r}}$ the node degree is $(r+1)$ and the diameter is $(2 r-2)$.

Hence the cost of $F_{r}$ is given by

$$
\begin{aligned}
\text { Cost } & =\text { degree } * \text { diameter } \\
& =(r+1)(2 r-2)
\end{aligned}
$$

Hence proved.

Proposition 3.8: Bisection Width of $\mathrm{F}_{\mathrm{r}}$ is $2^{m h-1}\left(1+2^{k-1}\right)$.

Proof: For a Metacube the bisection width is

$$
2^{m h-1}
$$

In Folded Metacube number of augmented edges is equal to $2^{m h+k-1}$

So bisection width is

$$
\begin{aligned}
& 2^{m h-1}+2^{m h+k-1} / 2 \\
& =2^{m h-1}\left(1+2^{k-1}\right)
\end{aligned}
$$

Hence the theorem is proved.

The topological properties of Folded Metacube are summarized in Table1.

\begin{tabular}{|l|l|l|l|l|}
\hline Network & Degree & Diameter & Cost & No. of edges \\
\hline HC & & & $n^{2}$ & $\mathrm{n} .2^{\mathrm{n}-1}$ \\
\hline FHC & $\mathrm{n}+1$ & $\lceil n / 2\rceil$ & $\left\lceil n / 2^{k}(\mathrm{n}+1)\right.$ & $(\mathrm{n}+1) 2^{\mathrm{n}-1}$ \\
\hline DC & $(\mathrm{n}+1) / 2$ & $\mathrm{n}+1$ & $(\mathrm{n}+1)^{2} / 2$ & $(\mathrm{n}+1) 2^{\mathrm{n}-2}$ \\
\hline MC & & $(\mathrm{m}+1) 2^{\mathrm{k}}$ & $\mathrm{r}(\mathrm{m}+1) 2^{\mathrm{k}}$ & $2^{m 2^{k}+k-1} r$ \\
\hline FMC & $\mathrm{r}+1$ & $2 \mathrm{r}-2$ & $(\mathrm{r}+1)(2 \mathrm{r}-2)$ & $2^{m 2^{k}+k-1}(r+1)$ \\
\hline
\end{tabular}




\section{ROUTING IN FMC NETWORK}

The routing in a cube based network depends upon shortest path, the Hamming distance. In $F_{r}$ the hamming distance is 1 or $(\mathrm{mh}+\mathrm{k})$. Two distinct algorithms for one-to-one and one-to-all communication in the FMC are proposed below.

\subsection{One-to-one Routing}

This algorithm performs the routing between any pair of nodes namely $\mathrm{u}, \mathrm{v} \in \mathrm{V}$ of $\mathrm{F}_{\mathrm{r}}$.

Algorithm One-to-one (a(u),a(v),r)

Begin

$$
a(w)=(a(u) \oplus a(v) ;
$$

If $\|a(w)\|<2 r-2$

Route the message sent from $u$ via a path composed of links with labels corresponding to bit position which are 1 's in $a(w)$

Else

send the message to $u^{\prime}$ via the complementary link, route the message via a path composed of links with labels corresponding to bit positions that are 0's in a(w).

End;

So the length of the shortest path in Folded Metacube is at most $(2 r-2)$, the diameter of the network.

\subsection{Broadcasting One-to-all}

The broadcasting process should satisfy the following desirable properties.

1. A node should not send (receive) the message to (from) more than one of its neighbors.

2. A node receives the message exactly once for the whole duration of the broadcasting.

Let $\mathrm{s}$ be source node in class 0 . Then $(\mathrm{r}+1)$ neighbors of $\mathrm{s}$ are $\mathrm{s}$, $0<\mathrm{i}<=\mathrm{r}+1$.

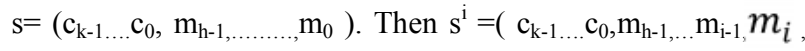
$\left.\mathrm{m}_{\mathrm{i}+1, \ldots \ldots,}, \mathrm{m}_{0}\right)$ where $0<\mathrm{i}<=\mathrm{r}-1$.

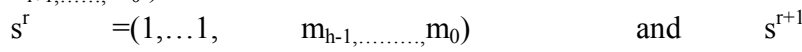

$=\left(c_{k-1}, \ldots c_{0}, m_{h-1}, \ldots . m_{0}\right)$.

Algorithm

1. Source $\mathrm{s}$ will send a message to its neighbor $\mathrm{s}^{\mathrm{r}}$ through cross edge.

2. Then $s$ and $s^{r}$ will broadcast simultaneously in their respective clusters using binomial trees.

3. Then a spanning broadcast tree (SBT) [4] can be constructed for $F_{r}$, where each node can be connected by cube edge if the node belongs to the same cluster.

4. If the next node belongs to a cluster of another class, then a cross edge is used. Next if the next node belongs to different cluster of same class then a complementary edge is used.
In SBT each node is connected by cube edge or a cross edge, if the hamming distance is less than $2 \mathrm{r}-2$, otherwise a complementary edge is used. So the height of the spanning broadcast tree is at most $2 \mathrm{r}-2$. Hence, the broadcasting is done in $2(\mathrm{~m}+\mathrm{k})-2$ time where as for Metacube it is $2^{\mathrm{k}}(\mathrm{m}+1)+\mathrm{k}-1$. The algorithm is illustrated below through a simple example.

\subsection{Illustration}

Let the distance between any two nodes $s$ and $t$ in $\operatorname{FMC}(2,2)$ be calculated. Then $\mathrm{mh}+\mathrm{k}=10$ bit node address of $\mathrm{s}$ be $(00,0000,0000)$. Let $t$ be the farthest node then its node address is $(00,1111,1111)$. Both the nodes $\mathrm{s}$ and $\mathrm{t}$ belong to class 0 but different clusters.

Node $s$ has five immediate neighbors. They are

$(00,0000,0001),(00,0000,0010),(10,0000,0000)$, $(01,0000,0000),(11,1111,1111)$

So path from $s$ to $t$ will be

$(00,0000,0000)->(11,1111,1111)->(10,1111,1111)-$ $>(00,1111,1111)$ as shown in Fig. (4).

And distance is 3 . But in $\mathrm{MC}(2,2)$ the distance between $\mathrm{s}$ and $\mathrm{t}$ is $(\mathrm{m}+1) 2^{\mathrm{k}}=12[11]$.

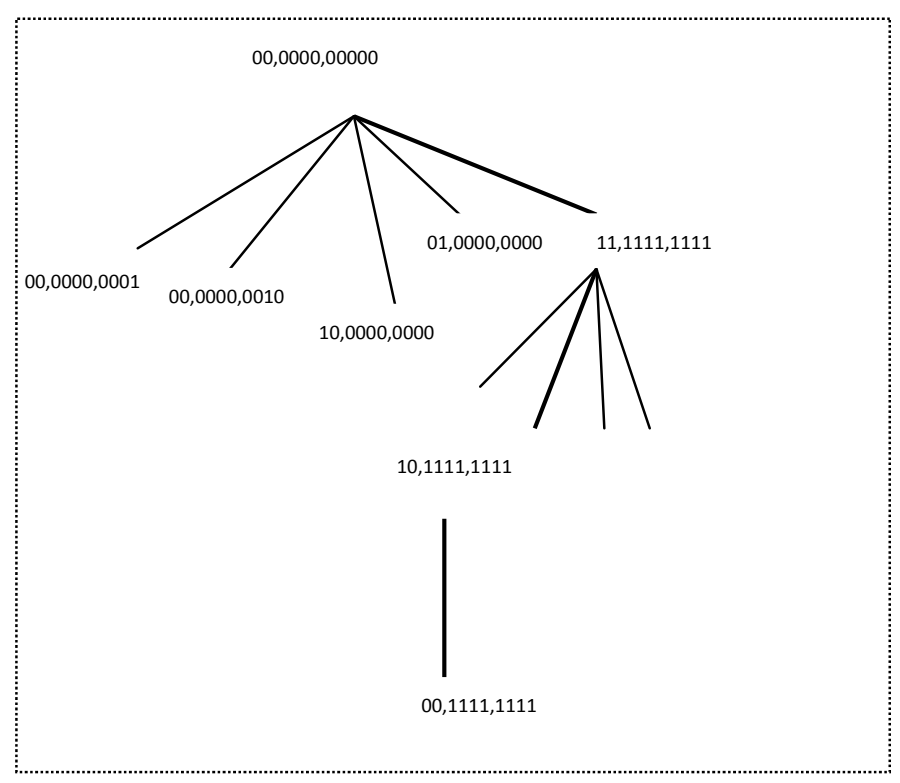

Figure 4. Spanning broadcast tree for $\operatorname{FMC}(2,2)$

\section{Performance Evaluation}

In this section, the performance of the Folded Metacube is evaluated and compared with some known cube based topologies. The various performance measures used for comparison include degree, diameter, cost, broadcasting time, cost effectiveness factor, time cost effectiveness factor. Reliability analysis is also done for the proposed network and compared with parent topologies.

\subsection{Cost Effectiveness Factor}

The cost effectiveness is one of the interesting criteria for cost performance measure $[13,14]$. This factor takes into account the cost of the entire multiprocessor as well as the processor utilization by the parallel algorithm under consideration. For 
evaluating the cost effectiveness of a parallel algorithm on a particular architecture, along with cost of processor, the cost of communication link is also considered. So cost effectiveness factor gives more insight to the performance of the multiprocessor system. Sometimes, two algorithms may have same speed-up on two different architectures. In such case the better choice will be the cheaper machine.

Theorem 5.1. The cost effectiveness factor of $F M C(k, m)$ is $\frac{1}{1+\rho\left(\frac{m+k+1}{2}\right)}$ ; where $\rho$ is the ratio of link to

processor cost.

Proof: The total number of processors in FMC is given by

$\mathrm{p}=2^{m h+k}$

The total number of edges is given by $E=(m+k+1) 2^{m h+k-1}$

$=(m+k+1) p / 2=f(p)$

So $g(p)=f(p) / p=(m+k+1) / 2$.

Hence the cost effectiveness factor is given by

$$
\operatorname{CEF}(p)=\frac{1}{1+\rho g(p)}=\frac{1}{1+\rho\left(\frac{m+k+1}{2}\right)}
$$

Hence the theorem is proved.

The cost effective analysis is done for both the networks FMC and $\mathrm{MC}$ for various dimensions. The computed values are presented in Table 2 and 3 for comparison. It is observed that for the same number of processors, the $C E F(p)$ of Folded Metacube is less than that of Metacube.

Table 2: Cost effectiveness factor of FMC

\begin{tabular}{|c|c|c|c|c|c|}
\hline $\boldsymbol{k}$ & $\mathrm{M}$ & $\boldsymbol{\rho}=0.1$ & $\boldsymbol{\rho}=0.2$ & $\boldsymbol{\rho}=0.3$ & $\boldsymbol{\rho}=0.4$ \\
\hline 1 & 2 & 0.833333 & 0.714286 & 0.625 & 0.555556 \\
\hline 1 & 3 & 0.8 & 0.666667 & 0.571429 & 0.5 \\
\hline 1 & 4 & 0.769231 & 0.625 & 0.526316 & 0.454545 \\
\hline 2 & 2 & 0.8 & 0.666667 & 0.571429 & 0.5 \\
\hline 2 & 3 & 0.769231 & 0.625 & 0.526316 & 0.454545 \\
\hline 2 & 4 & 0.740741 & 0.588235 & 0.487805 & 0.416667 \\
\hline 3 & 2 & 0.769231 & 0.625 & 0.526316 & 0.454545 \\
\hline 3 & 3 & 0.740741 & 0.588235 & 0.487805 & 0.416667 \\
\hline 3 & 4 & 0.714286 & 0.555556 & 0.454545 & 0.384615 \\
\hline
\end{tabular}

Table 3: Cost effectiveness factor of MC

\begin{tabular}{|r|r|r|r|r|r|}
\hline $\boldsymbol{k}$ & $\boldsymbol{m}$ & $\boldsymbol{\rho}=0.1$ & $\boldsymbol{\rho}=0.2$ & $\boldsymbol{\rho}=0.3$ & $\boldsymbol{\rho}=0.4$ \\
\hline 1 & 2 & 0.869565 & 0.689655 & 0.689655 & 0.625 \\
\hline 1 & 3 & 0.833333 & 0.714286 & 0.625 & 0.555556 \\
\hline 1 & 4 & 0.8 & 0.666667 & 0.571429 & 0.5 \\
\hline 2 & 2 & 0.833333 & 0.714286 & 0.625 & 0.555556 \\
\hline 2 & 3 & 0.8 & 0.666667 & 0.571429 & 0.5 \\
\hline 2 & 4 & 0.769231 & 0.625 & 0.526316 & 0.454545 \\
\hline 3 & 2 & 0.8 & 0.666667 & 0.571429 & 0.5 \\
\hline 3 & 3 & 0.769231 & 0.625 & 0.526316 & 0.454545 \\
\hline 3 & 4 & 0.740741 & 0.588235 & 0.487805 & 0.416667 \\
\hline
\end{tabular}

\subsection{Time-cost-effectiveness Factor}

The time-cost-effectiveness factor, (TCEF) is an important measure to consider the situation where, a faster solution to a problem is more rewarding than a slower solution [13]. In other words, a delayed solution reduces the benefit that is obtained from the results. Time cost effectiveness factor considers time for solution of a problem as a parameter for evaluating the performance.

For $\mathrm{FMC}(\mathrm{k}, \mathrm{m})$ TCEF is given by,

$$
\operatorname{TCEF}\left(p, T_{p}\right)=\frac{1+\sigma T_{1}^{\alpha-1}}{1+\rho g(p)+\frac{T_{1}^{\alpha-1} \sigma}{p}}
$$

where $T_{1}$ is the time required to solve the problem by a single processor using the fastest sequential algorithm, $T_{p}$ is the time required to solve the problem by a parallel algorithm using a multiprocessor system having p processors ahd $\sigma$ is the ratio of the cost of penalty to cost of processors. For linear time penalty in $T_{p}, \alpha$ is chosen as 1 . TCEF is computed against dimension for FMC network and also for MC Network and listed in Table 4 and 5 for comparison. It is observed that for the same number of processors the TCEF(p) of Folded Metacube is higher than that of Metacube. Thus solving a problem using FMC network will be financially profitable.

Table 4: Time Cost Effectiveness Factor of FMC with $\sigma=1$

\begin{tabular}{|r|r|r|l|l|l|}
\hline $\mathrm{K}$ & $\mathrm{M}$ & $\boldsymbol{\rho}=\mathbf{0 . 1}$ & $\boldsymbol{\rho}=\mathbf{0 . 2}$ & $\boldsymbol{\rho}=\mathbf{0 . 3}$ & $\boldsymbol{\rho}=0.4$ \\
\hline 1 & 2 & 1.69312169 & 1.50234742 & $\mathbf{1 . 3 5 0 2 1 0 9 7}$ & $\mathbf{1 . 2 2 6 0 5 3 6 4}$ \\
\hline 1 & 3 & 1.65588616 & 1.42064373 & 1.24392614 & $\mathbf{1 . 1 0 6 3 0 9 4 2}$ \\
\hline 1 & 4 & 1.53615362 & $\mathbf{1 . 2 4 8 4 7 5 9 8}$ & $\mathbf{1 . 0 5 1 5 5 0 6 3}$ & $\mathbf{0 . 9 0 8 2 8 4 5 5}$ \\
\hline 2 & 2 & 1.56742691 & $\mathbf{1 . 2 8 9 5 1 0 1 4}$ & $\mathbf{1 . 0 9 5 3 0 4 3 1}$ & $\mathbf{0 . 9 5 1 9 3 8 2 7}$ \\
\hline 2 & 3 & 1.4814145 & $\mathbf{1 . 1 7 6 4 2 8 3 5}$ & $\mathbf{0 . 9 7 5 5 8 0 7 1}$ & $\mathbf{0 . 8 3 3 3 1 2 1 4}$ \\
\hline 2 & 4 & 1.37930672 & $\mathbf{1 . 0 5 2 6 2 9 4 7}$ & $\mathbf{0 . 8 5 1 0 6 2 4 5}$ & $\mathbf{0 . 7 1 4 2 8 4 7 4}$ \\
\hline 3 & 2 & 1.44143946 & $\mathbf{1 . 1 2 6 7 5 9 3 5}$ & $\mathbf{0 . 9 2 4 8 5 4 6 8}$ & $\mathbf{0 . 7 8 4 3 1 3 1 4}$ \\
\hline 3 & 3 & 1.3559322 & $\mathbf{1 . 0 2 5 6 4 1 0 2}$ & $\mathbf{0 . 8 2 4 7 4 2 2 7}$ & $\mathbf{0 . 6 8 9 6 5 5 1 7}$ \\
\hline 3 & 4 & 1.26984127 & $\mathbf{0 . 9 3 0 2 3 2 5 6}$ & $\mathbf{0 . 7 3 3 9 4 4 9 5}$ & $\mathbf{0 . 6 0 6 0 6 0 6 1}$ \\
\hline
\end{tabular}


Table 5: Time Cost effectiveness factor of MC with $\sigma=1$

\begin{tabular}{|c|c|c|c|c|c|}
\hline k & m & $\sigma=0.1$ & $\sigma=0.2$ & $\sigma=0.3$ & $\sigma=0.4$ \\
\hline 1 & 2 & 1.624365 & 1.39738 & 1.226054 & 1.09215 \\
\hline 1 & 3 & 1.590062 & 1.326425 & 1.137778 & 0.996109 \\
\hline 1 & 4 & 1.536154 & 1.248476 & 1.051551 & 0.908285 \\
\hline 2 & 2 & 1.598751 & 1.332466 & 1.14222 & 0.999512 \\
\hline 2 & 3 & 1.538389 & 1.249952 & 1.052598 & 0.909066 \\
\hline 2 & 4 & 1.481477 & 1.176468 & 0.975608 & 0.833332 \\
\hline 3 & 2 & 1.538459 & 1.249999 & 1.052631 & 0.90909 \\
\hline 3 & 3 & 1.481481 & 1.176471 & 0.97561 & $\mathbf{0 . 8 3 3 3 3 3}$ \\
\hline 3 & 4 & 1.428571 & 1.111111 & 0.909091 & 0.769231 \\
\hline
\end{tabular}

\subsection{Reliability Analysis}

The reliability analysis is an important criterion to evaluate the robustness of a parallel interconnection network $[14,15,16]$. Terminal and Broadcast Reliability measures are the main two measures. For the current network, two terminal reliability measure is evaluated and compared. Terminal reliability is the probability of the existence of at least one fault free path between a designated pair of input and output terminals. The FMC being a directed graph, the vertices and edges are weighted with reliabilities of the components they represent. Two nodes $\mathrm{A}$ and $\mathrm{B}$ are considered with $\mathrm{n}$ number of node disjoint paths lying between them. Let $r_{i}$ be the number of links involved in path $\mathrm{i}$, where $1 \leq \mathrm{i} \leq \mathrm{n}$. Thus there are $\mathrm{r}_{\mathrm{i}}-1$ number of nodes in path $i$.

Let $P\left(E_{i}\right)$ be the probability of successful route through the $\mathrm{i}^{\text {th }}$ path.

Then $R_{l}$ be the link reliability with link failure rate $(\lambda)$ is 0.0002 and

$R_{n} \quad$ is the node reliability with processor failure rate $(\lambda)$ is 0.002 .

So $R_{l}=e^{-\lambda t}$, where $\lambda=0.0002$ and $\mathrm{t}=2000$ and $R_{n}=$ $e^{-\lambda t}$, where $\lambda=0.002$ and $\mathrm{t}=2000$.

Theorem 5.3.1: For $\mathrm{FMC}(\mathrm{k}, \mathrm{m})$ network the two terminal reliability is given by

$T R=1-\prod_{i=1}^{n}\left(1-R_{l}^{r_{i}} R_{n}^{r_{i}-1}\right)$.

Proof: All nodes and links are considered to be identical with their failure rates statistically independent and exponentially distributed. Now the probability of existence of a successful connection between the source and destination can be given by

$$
\begin{aligned}
& P\left(E_{i}\right)=R_{l}^{r_{i}} R_{n}^{r_{i}-1} \\
\text { So } \quad & T R=P\left(E_{l} \cup E_{2} \cup E_{3} \cup \ldots \cup E_{n}\right) \\
= & 1-\prod_{i=1}^{n}\left(1-R_{l}^{r_{i}} R_{n}^{r_{i}-1}\right) .
\end{aligned}
$$

Normally there are three possible cases. Namely i) only paths are reliable, ii) only nodes are reliable, iii) both nodes and paths are unreliable. For the current work case (iii) is considered for FMC and MC networks. The same reliability evaluation is also done keeping $r$ fixed at 3 with different values of $t$. Computed values are shown in Table 6 and 7 for comparison. For Folded Metacube network the number of node disjoint paths (ndp) is more than that of $\mathrm{MC}$ network. For $\mathrm{MC}$ it is equal to $\mathrm{r}$.

Table 6. Reliability analysis

\begin{tabular}{|c|c|c|c|c|c|}
\hline $\boldsymbol{k}$ & $\boldsymbol{m}$ & $\mathrm{n}$ & Ndp & FMC & MC \\
\hline 1 & 2 & 3 & 4 & $\mathbf{0 . 8 1 8 7 3 2}$ & $\mathbf{0 . 0 1 3 5 4 2}$ \\
\hline 1 & 3 & 4 & 5 & $\mathbf{0 . 8 1 8 7 3 2}$ & $\mathbf{0 . 0 1 4 7 5 6}$ \\
\hline 1 & 4 & 5 & 6 & $\mathbf{0 . 8 1 8 7 3 2}$ & $\mathbf{0 . 0 1 5 9 6 9}$ \\
\hline 2 & 2 & 4 & 5 & $\mathbf{0 . 8 1 8 7 3 2}$ & $\mathbf{0 . 0 1 4 7 5 6}$ \\
\hline 2 & $\mathbf{3}$ & 5 & 6 & $\mathbf{0 . 8 1 8 7 3 2}$ & $\mathbf{0 . 0 1 5 9 6 9}$ \\
\hline 2 & 4 & 6 & 7 & $\mathbf{0 . 8 1 8 7 3 2}$ & $\mathbf{0 . 0 1 7 1 8}$ \\
\hline 3 & 2 & 5 & 6 & $\mathbf{0 . 8 1 8 7 3 2}$ & $\mathbf{0 . 0 1 5 9 6 9}$ \\
\hline 3 & 3 & 6 & 7 & $\mathbf{0 . 8 1 8 7 3 3}$ & $\mathbf{0 . 0 1 7 1 8}$ \\
\hline 3 & 4 & 7 & 8 & $\mathbf{0 . 8 1 8 7 3 3}$ & $\mathbf{0 . 0 1 8 3 9}$ \\
\hline
\end{tabular}

Table 7. Two terminal reliability comparison with $r=3$ versus time

\begin{tabular}{|c|c|c|}
\hline $\begin{array}{c}\text { Time in } \\
\text { Hrs }\end{array}$ & FMC(k,m) & MC(k,m) \\
\hline 1000 & $\mathbf{0 . 0 8 4 7 6 4}$ & $\mathbf{0 . 0 1 1 0 5 3}$ \\
\hline 2000 & $\mathbf{0 . 0 0 8 2 1 1}$ & $4.1 \mathrm{E}-05$ \\
\hline 3000 & $\mathbf{0 . 0 0 0 7 4 7}$ & $\mathbf{1 . 5 2 E - 0 7}$ \\
\hline 4000 & $6.77 \mathrm{E}-05$ & $\mathbf{5 . 6 1 E - 1 0}$ \\
\hline 5000 & $\mathbf{6 . 1 4 E - 0 6}$ & $\mathbf{2 . 0 7 E - 1 2}$ \\
\hline 6000 & $\mathbf{5 . 5 7 E - 0 7}$ & $\mathbf{7 . 6 6 E - 1 5}$ \\
\hline 7000 & $\mathbf{5 . 0 6 E - 0 8}$ & $\mathbf{0}$ \\
\hline 8000 & $4.59 E-09$ & 0 \\
\hline 9000 & $4.16 E-10$ & 0 \\
\hline
\end{tabular}

\section{RESULTS AND DISCUSSIONS}

In this section different performance parameters of the FMC are evaluated and compared against those of the parent networks. The Fig. (5), depicts the variation of degree with network dimension. Due to the augmentation of complementary links, the degree of the FMC is observed to be slightly greater than that of Metacube but quite less than HC and FHC.

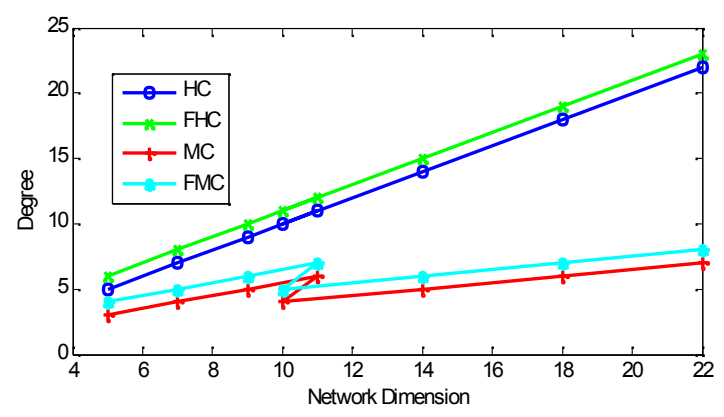

Figure 5. Comparison of Degree

Figure (6) shows the comparison of diameter with respect to dimension. It proves the superiority of the Folded Metacube over MC due to sufficient reduction in diameter. 


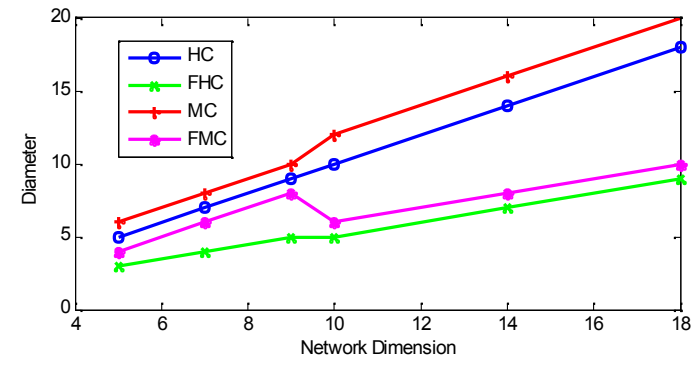

Figure 6. Comparison of diameter

Figure (7) presents comparison of cost among the four networks with respect to dimension. The cost of FMC is found to be smaller than that of Metacube. The cost is sufficiently reduced when number of nodes in the system is increased. The new network is found to have reduced cost while retaining higher packing density as compared to Hypercube and Folded Hypercube.

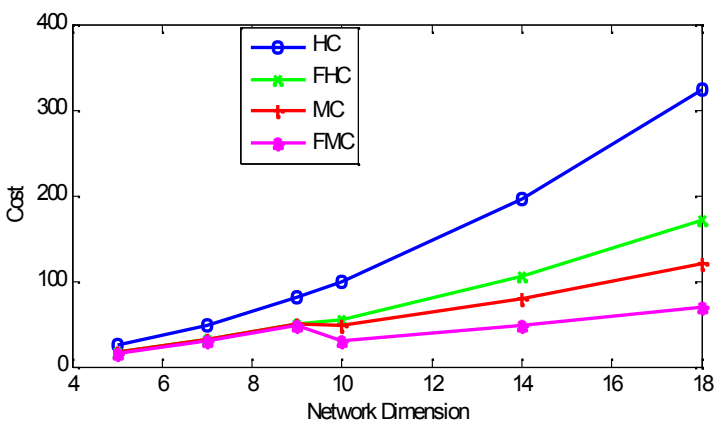

Figure 7: Cost comparison

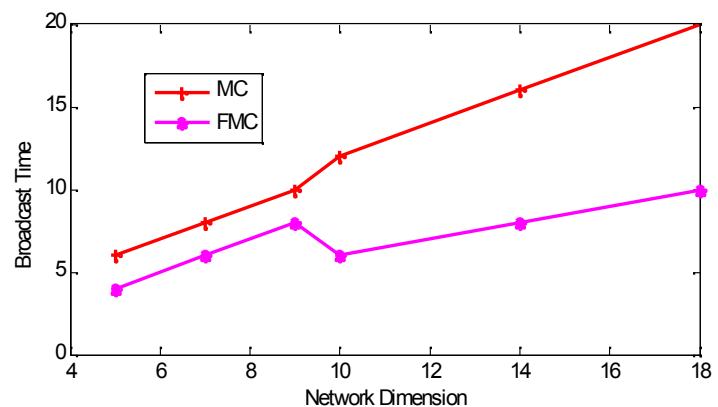

Figure 8: Broadcast Time versus Dimension

In Fig. (8), broadcast time of FMC is compared with that of Metacube with respect to dimension. Here $\mathrm{HC}$ and $\mathrm{FHC}$ are not taken into consideration, as with degree 3 the Hypercube and Folded hypercube both contain only 8 nodes and Metacube contains 32 nodes. The FMC exhibits quite a good improvement in broadcast time over its parent networks while connecting to millions of nodes.

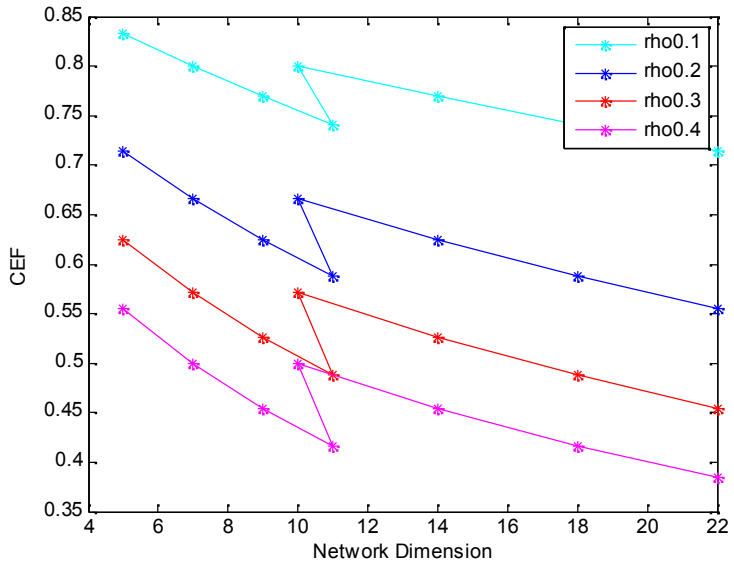

Figure 9: Cost effectiveness factor of FMC Network

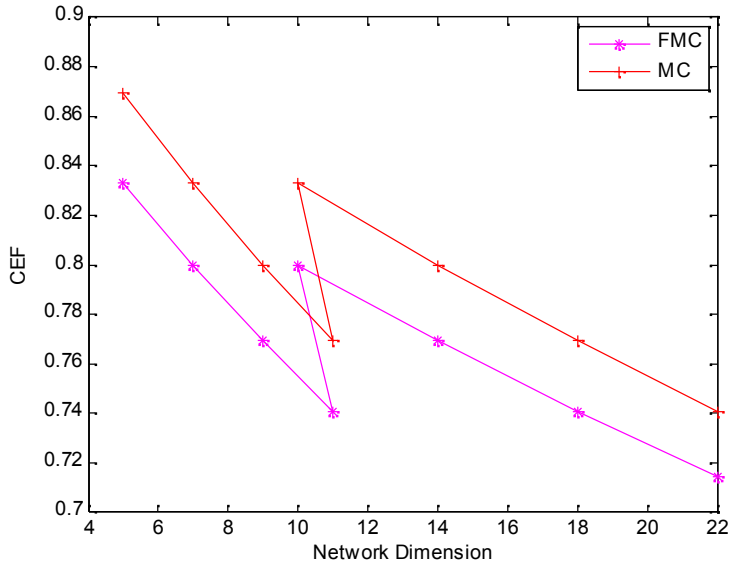

Figure10: CEF Comparison for MC and FMC network

Figure 9 shows the comparison of CEF for the proposed network with different values of $\rho$. It is a monotonically decreasing function like the Hypercube.

Also, it is observed that for the same number of processors, the $\mathrm{CEF}(\mathrm{p})$ of FMC network is quite less than that of the MC network as shown in Fig.10. Similarly, the TCEF values in Table 4 and 5 show that the FMC network will give faster solution to a problem. 


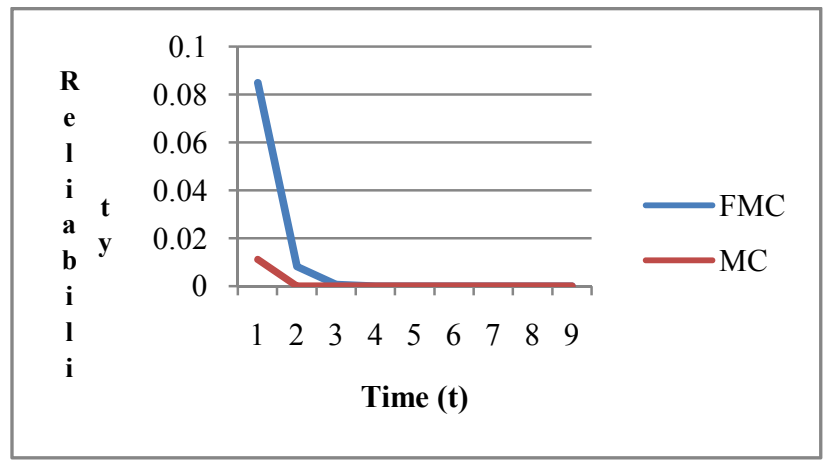

Figure 11: Comparison of reliability with time

Two terminal reliability computations listed in Table 6 and 7 show that Folded Metacube network is highly reliable when compared with Metacube network. The Fig. 11 shows the superiority of FMC over MC network. With increased degree, the FMC network possesses more node disjoint paths than MC network. Hence the two terminal reliability of FMC network is more than MC.

\section{CONCLUSION}

This paper introduces a new interconnection topology called Folded Metacube. The basic properties of Folded Metacube are derived and compared with its parent networks. Two routing algorithms are proposed for this new topology with lesser time complexities. The cost of the proposed topology is found to be less. This proposed topology is found superior to the parent networks in terms of reliability, cost effectiveness and time cost effectiveness. The reduced diameter helps to speed up the overall operation of large scale parallel systems. So the performance improvement will pay off the hardware overhead due to increase in node degree. With improved broadcast time the proposed network is a better candidate for large scale parallel systems which provides better performance and efficient inter processor communication.

\section{REFERENCES}

[1] N. Adhikari and C. R. Tripathy, "The Folded Crossed Cube: A New Interconnection Network for Parallel Systems, International Journal of Computer Applications, (2010), Vol.4, No.3, pp.44-50.

[2] N. Adhikari and C. R. Tripathy, "Folded Dualcube : A New Interconnection For Parallel Systems", Proceedings of the $11^{\text {th }}$ International Conference on Information Technology, India, 17-18 Dec. (2008, IEEE Computer Society) pp-75-78,

[3] N. Adhikari and C.R. Tripathy, "Folded Metacube : An Efficient Large Scale Parallel Interconnection Network", Proceedings of IEEE International Advance Computing Conference (IACC2009) , 6-7 March, ( IEEE Comp. Society, 2009) pp. 1281-1284.
[4] Ahmed EI-Amawy and Shahram Latifi, "Properties and Performance of Folded Hypercubes", IEEE Transactions on Parallel and Distributed Systems, vol. 2, No.1, Jan1991, pp. 31-42.

[5] A. Avizienis, “Fault Tolerant System”, IEEE Transactions on Computers, vol. 25, no 12, 1976, pp.1304-1312 .

[6] L.N. Bhuyan And D. P. Agrawal, "Performance Of Multiprocessor Interconnection Network"; IEEE Computers, (1989).

[7] L. N. Bhuyan and D. P. Agarwal, "Generalized Hypercube and Hyperbus Structures For a Computer Network", IEEE Tran. On Computers, C-33, No. 4, April 1984, pp-323-333.

[8] K. Effe, " The Crossed Cube Architecture for Parallel Computation", IEEE Transactions on Parallel and Distributed Systems, (1992) Vol.3(5), pp. 513-524.

[9] T. Feng, “A survey Of Interconnection Networks", IEEE Computers, 1(4), 1981,pp.12-27,.

[10] Yamin Li, Shietung Peng and Wanming Chu, "Efficient Collective Communications in Dual-cube", The Journal of Super Computing, 28, (2004), pp.71-90.

[11] Yamin Li, Shietung Peng and Wanming Chu, "Metacube: A New Interconnection Network for Large Parallel System"; ACSAC02, Australian Computer Science Communications, vol.24(4), (2001), pp.29-36.

[12] Y. Saad and M.H. Schultz, " Topological properties of hypercubes", IEEE Transactions on Computers, (1988), Vol.37(9),pp. 867-872.

[13]D. Sarkar, "Cost and Time Cost Effectiveness of Multiprocessing", IEEE Transaction on Parallel and Distributed Systems, vol-5 No.4, pp704-712, June (1993).

[14] C.R. Tripathy, “ Star-cube: A New Fault Tolerant Interconnection Topology For Massively Parallel Systems", IE(I) Journal, ETE Div., vol.84, no 2, Jan (2004), pp.8392.

[15] C. R. Tripathy, R. N. Mohapatra and R. B. Mohapatra, “ Reliability Analysis of Hypercube Multicomputers", Microelectronics and Reliability- an International Journal, (1997) vol. 37(6), p.885

[16] A. Verma and C. S. Raghavendra, "Reliabilty Analysis of Redundant-Path Interconnection Networks", IEEE Transactions on Reliability, Vol. 38, No. 1, April( 1989). 